\title{
Disnea e impacto en la calidad de vida de los pacientes COVID-19 después del alta hospitalaria
}

\author{
Dyspnea and impact on the quality of life of COVID-19 patients after hospital discharge \\ Dispneia e impacto na qualidade de vida de pacientes com COVID-19 após alta hospitalar
}

\author{
Tania Abril Mera \\ tania.abril@cu.ucsg.edu.ec \\ ORCID: 0000-0003-0214-3518 \\ Luis Morán Luna \\ luis.moran03@cu.ucsg.edu.ec \\ ORCID: 0000-0003-1000-426X
}

\author{
Gianna Guzmán Menéndez \\ gianna.guzman@cu.ucsg.edu.ec \\ ORCID: 0000-0002-8114-1926 \\ Layla De la Torre Ortega \\ layla.delatorre@cu.ucsg.edu.ec \\ ORCID: 0000-0002-4813-6957
}

Universidad Católica de Santiago de Guayaquil, Ecuador

\begin{abstract}
RESUMEN
Introducción: El COVID-19 es una enfermedad infecciosa que puede causar disfunciones respiratorias y físicas a corto y largo plazo. La disnea como consecuencia de la fibrosis pulmonar progresa a medida que la enfermedad avanza y afecta la calidad de vida. Objetivo: Determinar el nivel de disnea y su impacto en la calidad de vida de los pacientes post COVID-19 después del alta hospitalaria. Metodología: Enfoque cuantitativo, alcance descriptivo relacional, diseño no experimental con una muestra de 82 pacientes de 20 a 65 años de edad, pertenecientes al Hospital Monte Sinaí de la ciudad de Guayaquil. Resultados: El mayor porcentaje de la población evaluada pertenecía al sexo masculino y las edades entre 50 a 59 años, donde la hipertensión sobresale entre las patologías pre-existentes; el $71 \%$ de los pacientes evaluados tiene disminución de la capacidad física, 1 a 4 en la escala de Borg; el $30 \%$ de los pacientes posee disnea moderada, el $35 \%$ indicó que su calidad de vida relacionada con la salud, ha tenido un impacto alto previo alta hospitalaria y se comprobó su dependencia entre el CAT y la mMRC, ya que aquellos pacientes con disnea grado 3 y 4 presentaban un impacto alto y muy alto en su calidad de vida y en el $70 \%$ de los pacientes evaluados la fatiga interfiere en sus actividades generales. Conclusión: Los pacientes Post COVID19 presentan disnea moderada que interfiere en su calidad de vida, las mismas que están relacionadas a la edad, antecedentes patológicos y el tiempo de evolución.
\end{abstract}

Palabras clave: COVID-19; disnea; calidad de vida; Fisioterapia; disfunciones respiratorias

\begin{abstract}
Introduction: COVID-19 is an infectious disease that can cause respiratory and physical dysfunctions in the short and long term. Dyspnea as a consequence of pulmonary fibrosis progresses as the disease progresses and affects quality of life. Objective: To determine the level of dyspnea and its impact on the quality of life of post-COVID-19 patients after hospital discharge. Methodology: Quantitative approach, relational descriptive scope, non-experimental design with a sample of 82 patients from 20 to 65 years of age, belonging to the Monte Sinaí Hospital in the city of Guayaquil. Results: The highest percentage of the evaluated population belonged to the male sex and the ages between 50 to 59 years, where hypertension stands out among the pre-existing pathologies; $71 \%$ of the patients evaluated have decreased physical capacity, 1 to 4 on the Borg scale; $30 \%$ of the patients have moderate dyspnea, $35 \%$ indicated that their health-related quality of life has had a high impact prior to discharge from hospital and their dependence between CAT and MMRC was confirmed, since those patients with dyspnea Grade 3 and 4 had a high and very high impact on their quality of life and in $70 \%$ of the patients evaluated, fatigue interferes with their general activities. Conclusion: Post COVID-19 patients present moderate dyspnea that interferes with their quality of life, which are related to age, pathological history and time of evolution.
\end{abstract}

TM: Licenciada en Terapia Física. Diplomado Superior en Pedagogía Universitaria. Magister en Educación Superior. Doctoranda en Medicina y Salud Pública de la Universidad de Granada España. Fisioterapeuta CRM\#2 INNFA. Docente y Coordinadora de admisión de carrera de Fisioterapia de la Universidad Católica de Santiago de Guayaquil. Directora del Centro de Rehabilitación Directora del Centro de Rehabilitación
Fisiovidaec. Universidad Católica De Santiago De Guayaquil. Ecuador.

GG: Licenciada en Terapia Física. Pregrado. Actualmente Fisioterapeuta del consultorio Fisio-Center. Universidad Católica Santiago de Guayaquil. Ecuador.

LM: Licenciada en Terapia Física. Pregrado. Actualmente Fisioterapeuta del consultorio Fisio-Center. Universidad Católica De Santiago De Guayaquil. Ecuador.

LD: Especialista en Fisioterapia en el Adulto Crítico. Licenciada en Terapia Física y Rehabilitación. Magister en Educación Superior. Experiencia Laboral: Docente de la Universidad Católica de Santiago de Guayaquil (UCSG), Fisioterapeuta Hospital General Guasmo Sur, Hospital Universitario de Guayaquil. Reconocimientos por Trabajo académico por el G.A.D de Laurel y UCSG Universidad Católica De Santiago De Guayaquil. Ecuador.

Key words: COVID-19; dyspnea; quality of life; Physiotherapy; respiratory dysfunctions 
TM: Licenciada en Terapia Física Diplomado Superior en Pedagogía Universitaria. Magister en Educación Superior. Doctoranda en Medicina y Salud Pública de la Universidad de Granada España. Fisioterapeuta CRM\#2 INNFA Docente y Coordinadora de admisión de carrera de Fisioterapia de la Universidad Católica de Santiago de Guayaquil. Directora del Centro de Rehabilitación Fisiovidaec. Universidad Católica De Santiago De Guayaquil. Ecuador.

GG: Licenciada en Terapia Física. Pregrado. Actualmente Fisioterapeuta del consultorio Fisio-Center. Universidad Católica Santiago de Guayaquil. Ecuador.

LM: Licenciada en Terapia Física. Pregrado. Actualmente Fisioterapeuta del consultorio Fisio-Center. Universidad Católica De Santiago De Guayaquil. Ecuador.

LD: Especialista en Fisioterapia en el Adulto Crítico. Licenciada en Terapia Física y Rehabilitación. Magister en Educación Superior. Experiencia Laboral: Docente de la Universidad Católica de Santiago de Guayaquil (UCSG), Fisioterapeuta Hospital General Guasmo Sur, Hospital Universitario de Guayaquil. Reconocimientos por Trabajo académico por el G.A.D de Laurel y UCSG Universidad Católica De Santiago De Guayaquil. Ecuador.

\section{RESUMO}

Introdução: COVID-19 é uma doença infecciosa que pode causar disfunções respiratórias e físicas em curto e longo prazo. A dispneia como consequência da fibrose pulmonar progride com a progressão da doença e afeta a qualidade de vida. Objetivo: Determinar o grau de dispneia e seu impacto na qualidade de vida de pacientes pós-COVID-19 após a alta hospitalar. Metodologia: Abordagem quantitativa, âmbito descritivo relacional, delineamento não experimental com amostra de 82 pacientes de 20 a 65 anos, pertencentes ao Hospital Monte Sinaí na cidade de Guayaquil. Resultados: O maior percentual da população avaliada pertencia ao sexo masculino e na faixa etária de 50 a 59 anos, onde a hipertensão se destaca entre as patologias pré-existentes; $71 \%$ dos pacientes avaliados têm capacidade física diminuída, 1 a 4 na escala de Borg; $30 \%$ dos pacientes apresentam dispneia moderada, 35\% indicaram que sua qualidade de vida relacionada à saúde teve um alto impacto antes da alta hospitalar e sua dependência entre CAT e mMRC foi confirmada, uma vez que aqueles pacientes com dispneia Os graus 3 e 4 tiveram um impacto alto e muito alto em sua qualidade de vida e em $70 \%$ dos pacientes avaliados, a fadiga interfere em suas atividades gerais. Conclusão: Pacientes pós COVID-19 apresentam dispneia moderada que interfere em sua qualidade de vida, que estão relacionados à idade, história patológica e tempo de evolução.

Palavras-chave: COVID-19; dispneia; qualidade de vida; fisioterapia; disfunções respiratorias

\section{INTRODUCCIÓN}

$\mathrm{E}$ 1 primer semestre del año 2020 se ha caracterizado por la presencia del virus SARS-CoV2 de la familia coronavirus, cuya sepa COVID-19 ha infectado hasta el mes de septiembre del 2020 la cantidad de 30,907,717 millones de personas en todo el mundo (1). En el Ecuador hasta la segunda semana de septiembre, se registraron 125.620 casos confirmados con pruebas PCR y 7.288 fallecidos (2).

Las personas contagiadas que alcanzan niveles de alta gravedad, son pacientes con disfunción pulmonar y respiratoria después del alta hospitalaria, debida al daño alveolar difuso, membranas hialinas ricas en fibrina y la activación de macrófagos producidas por el virus lo que contribuye al deterioro físico con deterioro de la capacidad para transferencias, disnea de esfuerzo, atrofia muscular, incluida la musculatura respiratoria, del tronco y de las extremidades además del estrés postraumático (3). Los mismos que son susceptibles a evaluación, seguimiento y tratamiento Fisioterapéutico, debido a las complicaciones propias de la enfermedad que afecta la funcionabilidad respiratoria y física derivadas del encamamiento prolongado.

La disnea como consecuencia de la fibrosis pulmonar que causa el COVID-19 progresa a medida que la enfermedad avanza y afecta a la calidad de vida del paciente durante y después del COVID; por lo que se debe tener especial precaución en pacientes con comorbilidad tal como hipertensión pulmonar, miocarditis, insuficiencia cardíaca, trombosis venosa profunda y fracturas inestables (4). El fisioterapeuta cumple un rol muy importante en todas las fases del COVID-19y la tele rehabilitación es una de las oportunidades científico-tecnológicas, para desarrollar protocolos de prevención, evaluación e intervención, dirigido a la 
población vulnerable y que por medidas de bioseguridad no puede asistir a un centro de Fisioterapia.

En Ecuador, el Hospital General Monte Sinaí fue considerado como centro de referencia para COVID-19 y viene atendiendo una importante cantidad de pacientes quienes luego del alta hospitalaria presentaron disminución de su calidad de vida debido a la persistencia de la disnea, síntoma frecuente en el COVID-19, además de otras alteraciones de tipo osteomusculares como debilidad, atrofia y parestesias.

El objetivo de esta investigación consistió en determinar el nivel de disnea y su impacto en la calidad de vida de los pacientes post COVID-19 después del alta hospitalaria, cuya vía de evaluación fue la tele-rehabilitación mediante la aplicación de WhatsApp y link de evaluación.

La pandemia del Covid-19 no solo ha causado un gran impacto social, económico y sanitario en el mundo, sino que también ha provocado cambios radicales en los hábitos y estilo de vida de las personas, principalmente aquellas que han padecido un cuadro respiratorio grave con hospitalización (5).

La presencia del fisioterapeuta ha sido fundamental dentro del equipo interdisciplinario de primera línea y después de la hospitalización de los pacientes infectados, con este artículo se pretende delimitar las secuelas que esta enfermedad puede dejar a nivel de la función pulmonar y de su capacidad física, además de su impacto en la calidad de vida, mediante instrumentos de fácil aplicación que permitan establecer un abordaje apropiado que satisfaga las necesidades del paciente.

\section{MATERIALES Y MÉTODOS}

S e realizó un estudio de enfoque cuantitativo, alcance descriptivorelacional, con diseño de tipo no experimental y de corte transversal; el universo poblacional estuvo conformado por 104 pacientes y la muestra por 82 pacientes con edades comprendidas de 20 a 65 años, los mismos que fueron internados entre los meses de mayo a junio del 2020 y dados de alta con diagnóstico de COVID-19 del Hospital General Monte Sinaí. La técnica empleada fue observacional, documentales y estadísticas como el Microsoft Excel y el software SPSS para relacionar y validar la hipótesis a través del Rho de Spearman. Referente a la evaluación tuvo un tiempo de duración de 3 meses de mayo a julio 2020 y se orientó de forma objetiva por medio de video-llamada WhatsApp y subjetiva por medio de un link de evaluación. Entre las variables figuraron: edad, sexo, capacidad aeróbica, disnea y calidad de vida en salud. Los criterios de inclusión que se consideraron fueron: pacientes de ambos sexos, en edades de 20 a 65 años, con historia clínica completa y diagnosticada con COVID-19 post hospitalización. Los instrumentos de evaluación en fisioterapia empleados fueron: Historia clínica, la escala de Borg, la Escala mMRC encargada de cuantificar la Disnea, el test Sit to Stand que mide la capacidad aeróbica del paciente, el inventario breve de la fatiga IBF y el cuestionario de CAT, que ha sido desarrollado para ayudar a pacientes $y$ médicos a evaluar el impacto que posee la calidad de vida en relación con la salud. 


\section{RESULTADOS}

$\mathrm{D}$

e acuerdo con la información analizada a través de las historias clínicas el mayor porcentaje de pacientes con secuelas post COVID-19 la tiene el sexo masculino con un $61 \%$, las edades de 50 a 59 años con un 18\% de la población y de 60 a 69 años con un $11 \%$, mientras que las mujeres entre 40 a 39 representan solo $7 \%$, a diferencia del $11 \%$ que comprometen edades de 50 a 59 y 60 a 69 años de edad. (Figura 1).

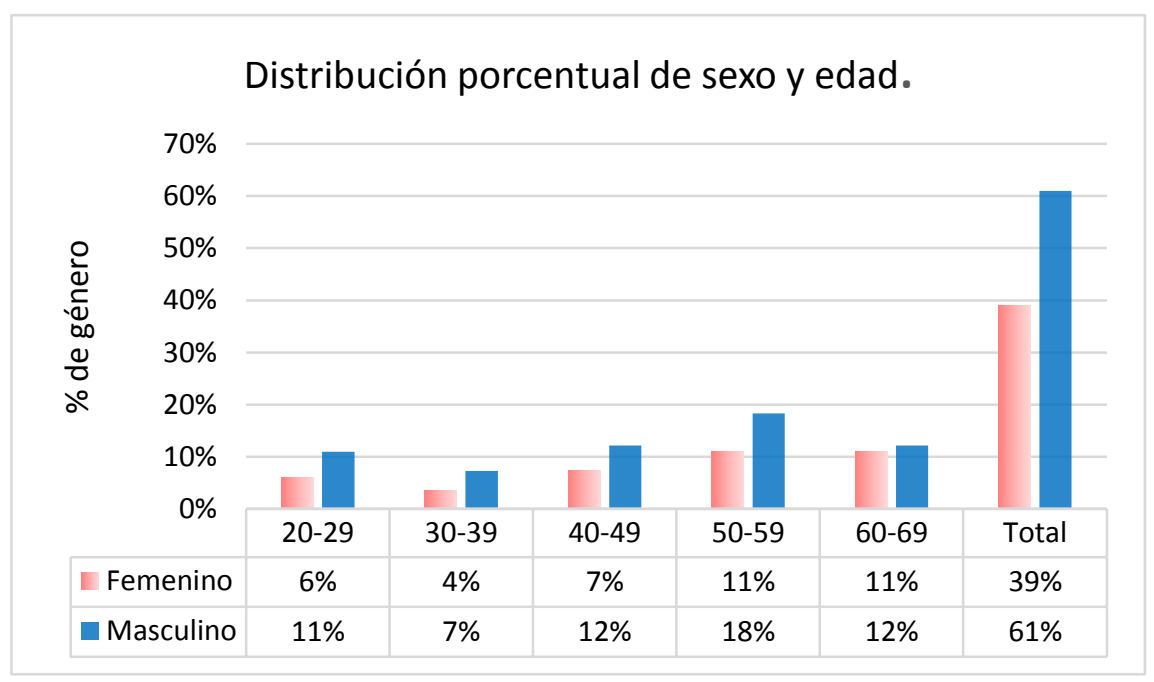

Figura 1. Distribución porcentual de sexo y edad.

De acuerdo a los datos analizados se logró identificar que, de la población evaluada, el $38 \%$ de los pacientes son hipertensos, la insuficiencia renal se encuentra con el $24 \%$ seguida de la diabetes con un $18 \%$, pese los valores evidenciados también se pudo determinar que el $28 \%$ de los pacientes no posee ninguna patología. (Figura 2).

Distribución porcentual antecedentes patológicos.

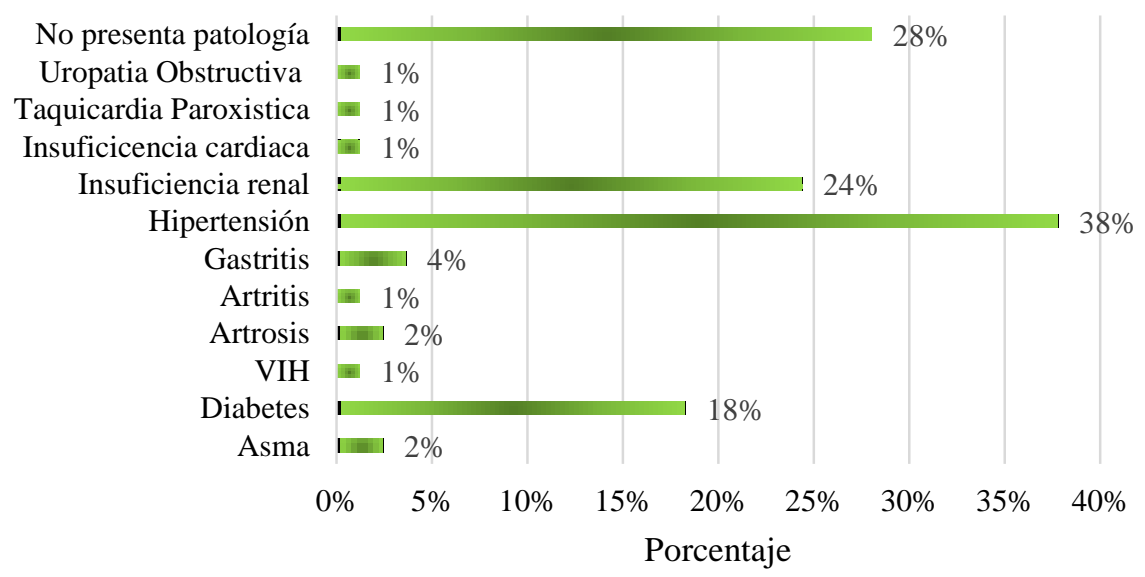

Figura 2. Distribución porcentual antecedentes patológicos. 
De acuerdo con el análisis a la información obtenida, el 16\% de la población evaluada manifestó grado 0 sin problemas de falta de aire, el $24 \%$ de los pacientes presentaron grado 1 de disnea cuando caminan de prisa, por otra parte, el grado 2 con 30\% indica la incapacidad que posee la persona para mantener el paso con la misma edad, a diferencia del grado 3 y 4 que representan un 15\% cada uno, presentando disnea al recorrer 100 metros e incapacidad de realizar las actividades de la vida diaria. (Figura 3).

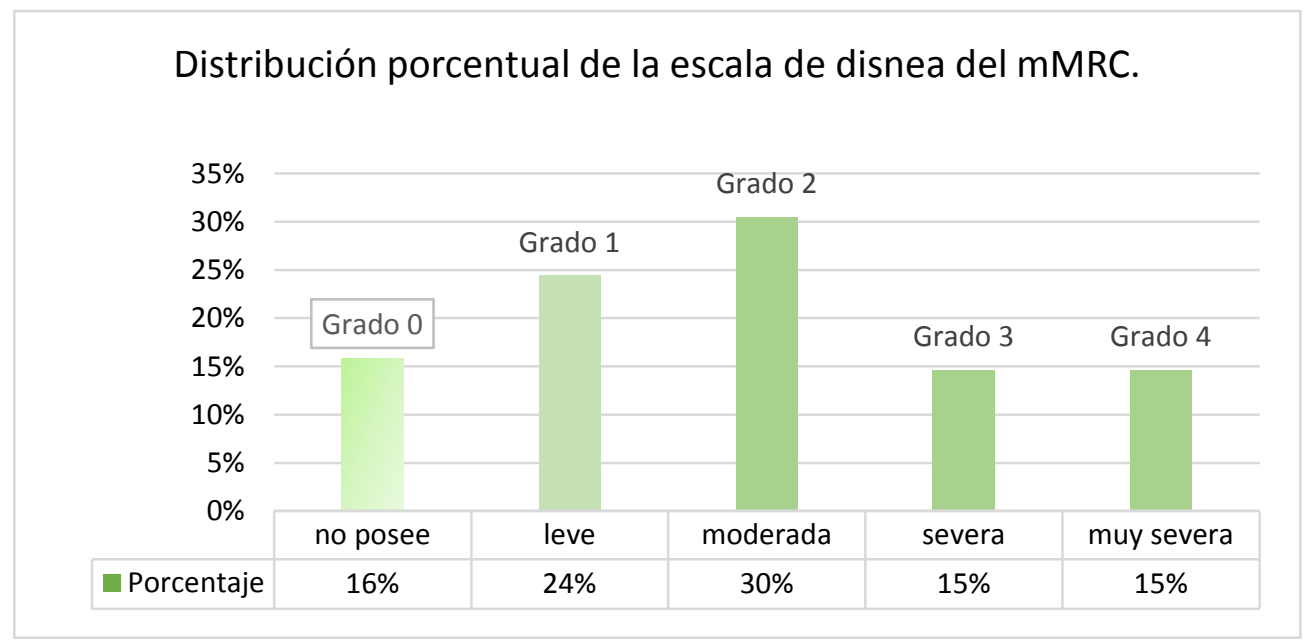

Figura 3. Distribución porcentual de la escala de disnea del mMRC.

De acuerdo a los resultados obtenidos de la escala de Borg, el 31\% de la población refirió tener un cansancio leve con una puntuación de1 y 2, el 32\% un cansancio percibido moderado con una puntuación de 3 y 4, cansancio grave lo obtuvo el 31\% de la población evaluada con puntuaciones de 5 a 7 y solo el $1 \%$ manifestó un cansancio grave. (Figura 4).

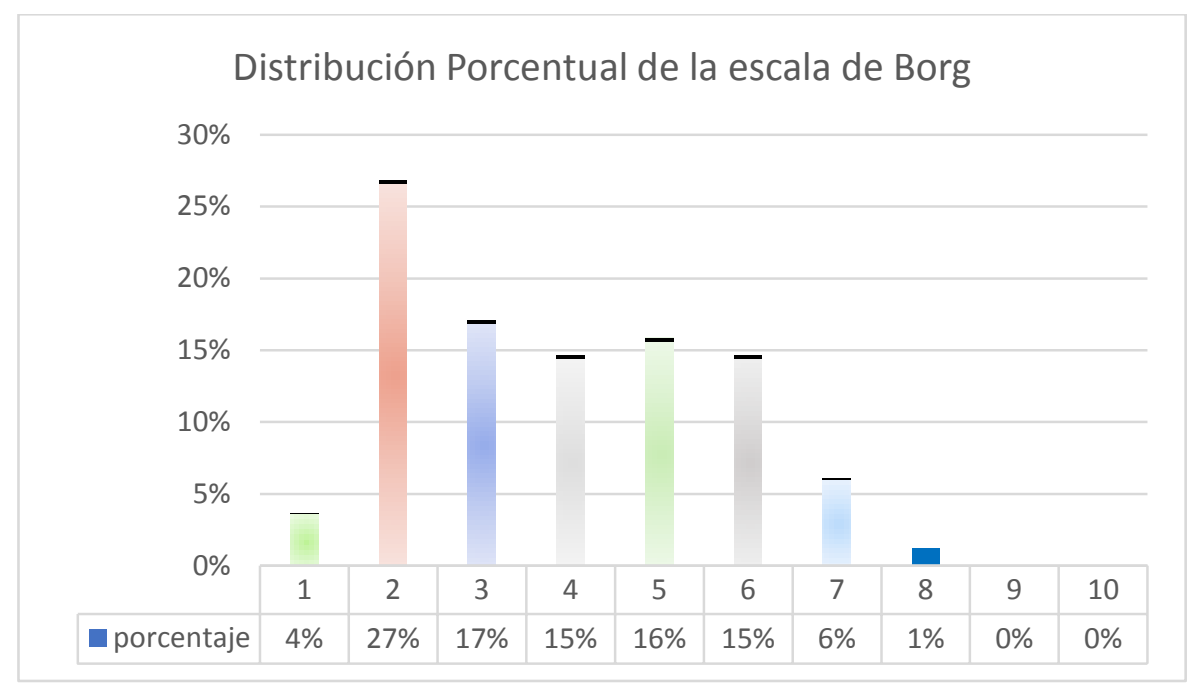

Figura 4. Escala de Borg. 
De acuerdo a la información obtenida y el 71\% de los pacientes realizó repeticiones inferiores de 11 veces, a diferencia del 29\% que pudo realizar de 12 a17 veces, mientras que mayor de 18 repeticiones no hubo registro. (Figura 5).

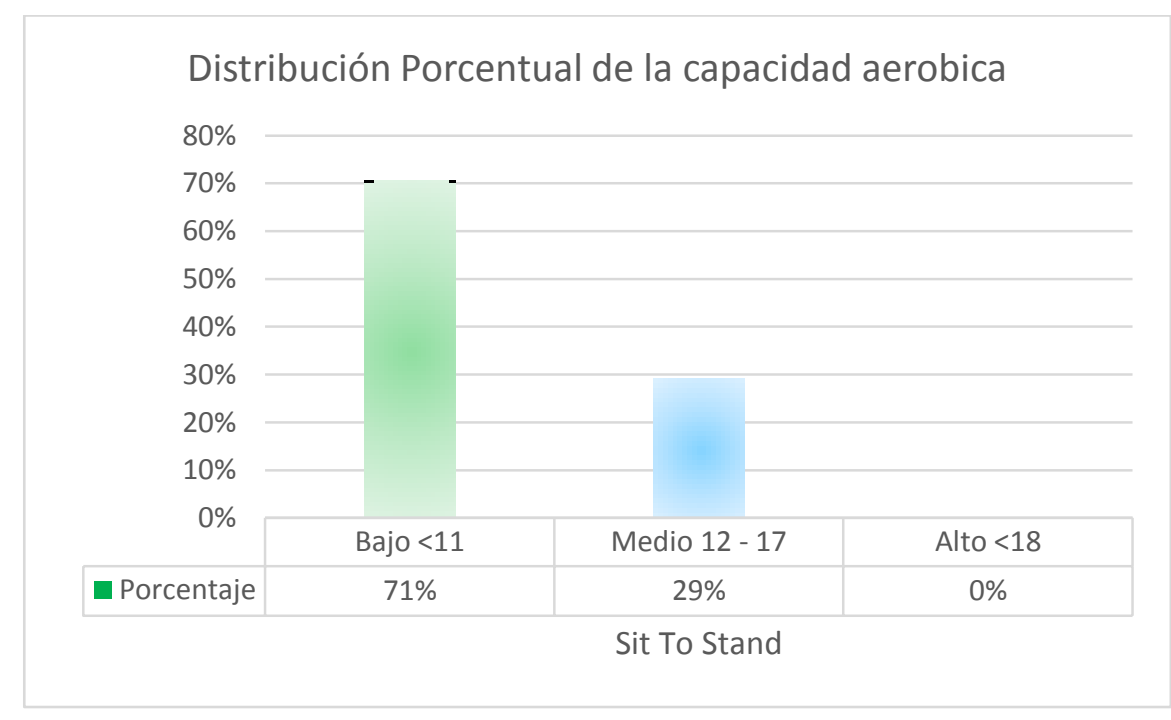

Figura 5. Distribución Porcentual del Test Sit To Stand.

De acuerdo con la información obtenida, se identifica que la fatiga en el 70\% de los pacientes evaluados infiere en sus actividades generales, respecto al estado de ánimo solo el 68\% expreso que infiere, mientras que la capacidad para caminar y el trabajo normal la fatiga infiere en el $61 \%$ y en las relaciones interpersonales los encuestados mencionaron un $70 \%$. (Figura 6).

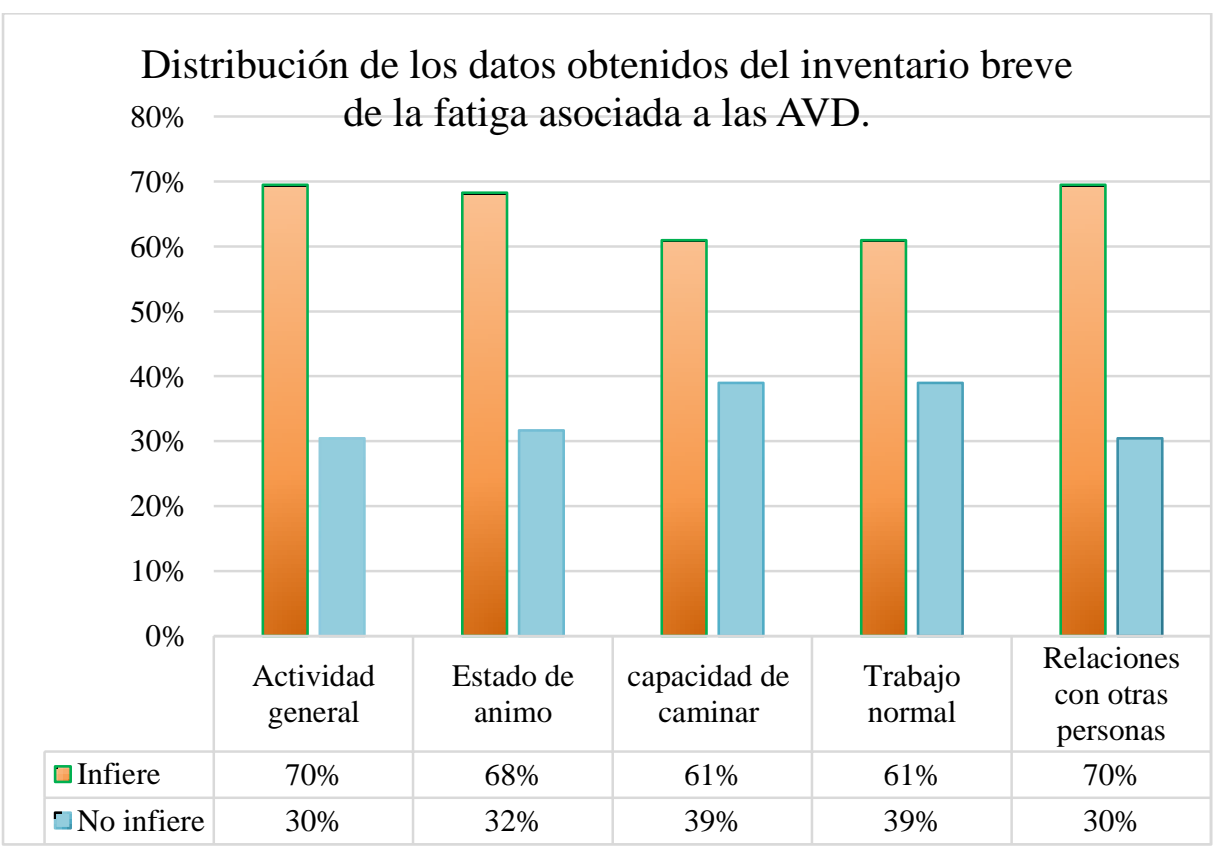

Figura 6. Distribución de los datos obtenidos del Inventario breve de la fatiga asociada a las AVD. 
De acuerdo con la información obtenida respecto a la calidad de vida en relación con la salud, que incluyen parámetros como tos, flema, opresión torácica, limitación de actividades domésticas, calidad del sueño y energía. Indica que el $28 \%$ de la población tiene un bajo impacto con ciertas limitaciones en sus actividades de la vida diaria, el $32 \%$ con un impacto medio, $35 \%$ con un impacto alto, a diferencia del $5 \%$ que limita las actividades de la vida diaria con un impacto muy alto que se relaciona a la calidad de vida del paciente post COVID-19. (Figura 7).

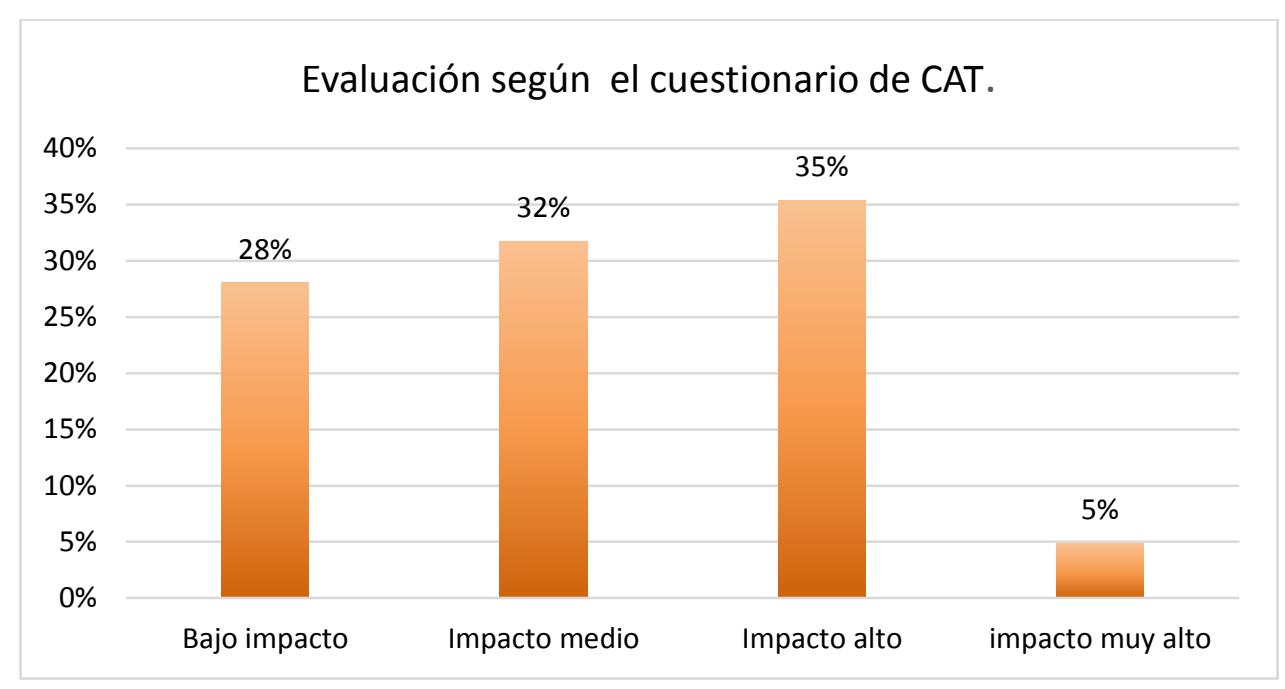

Figura 7. Evaluación según el cuestionario de CAT.

Se aprecia el grado de relación entre la Disnea y calidad de vida, por el Rho de Spearman $\rho=0,740$, respecto al análisis de significancia estadísticas tenemos un valor $\mathrm{p}=$ $0<0,05$ (95\% de confianza); por lo tanto, existe evidencia suficiente para rechazar la hipótesis nula, es decir hay evidencia suficiente para afirmar que la correlación es distinta de 0 , en conclusión, existe una correlación significativa entre ambas variables. Consiguientemente se detecta una correlación lineal positiva (directa). (Figura 8). 


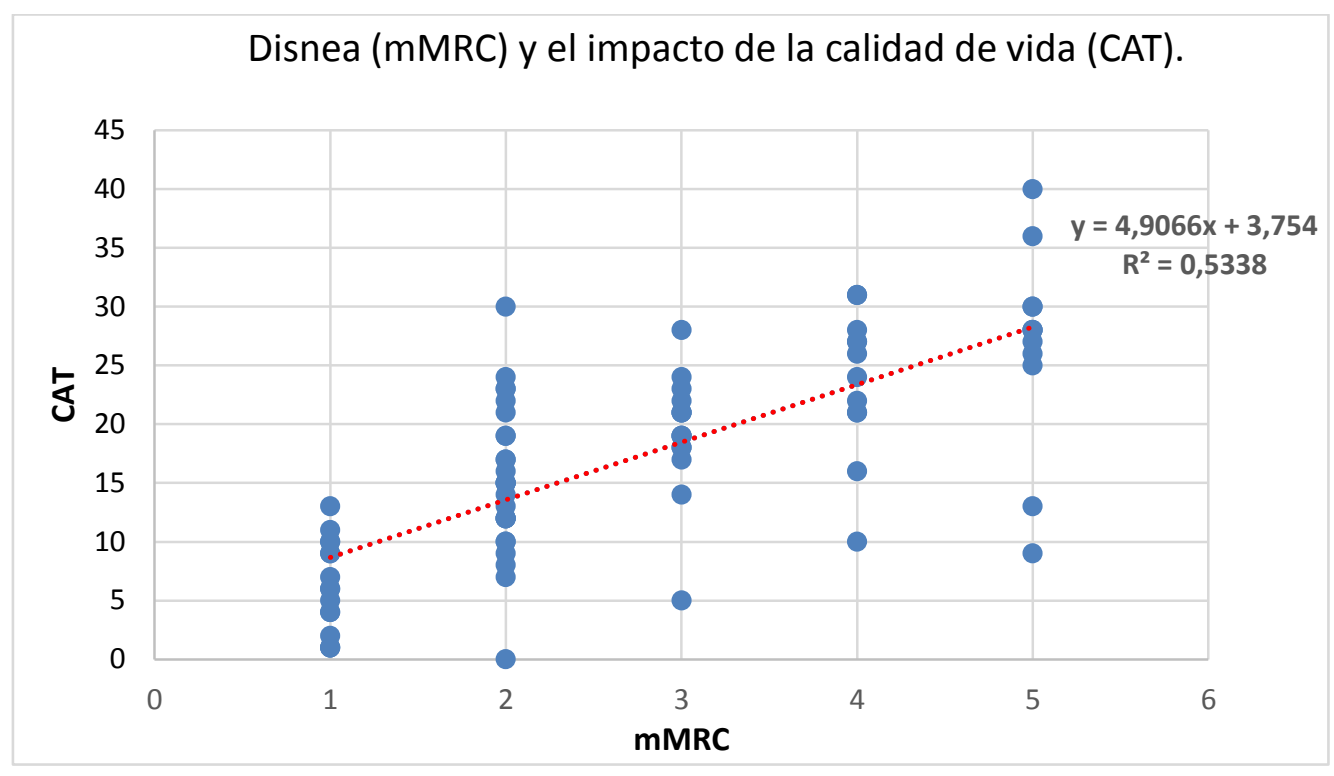

Figura 8. Disnea (mMRC) y el impacto de la calidad de vida (CAT).

Es decir, cuando aumenta el grado de disnea (moderada, severa y muy severa) el impacto de calidad de vida también aumenta (impacto medio, impacto alto y muy alto), de la misma forma si el grado de disnea es bajo (leve) el impacto de calidad de vida también será bajo.

Por medio de la aplicación de WhatsApp y link de evaluación, se verificó que la población evaluada en su mayoría era de sexo masculino entre 50 a 59 años de edad, donde la hipertensión sobresale entre las patologías pre-existentes; el 71\% de los pacientes evaluados tienen un índice bajo en la capacidad aeróbica (Sit to stand) y de 1 a 4 en la escala de Borg; el 30\% de los pacientes posee grado 2 (moderada) de disnea, en cuanto al grado de fatiga, en el $70 \%$ de los pacientes evaluados la fatiga interfiere en sus actividades generales, el $35 \%$ indicó que su calidad de vida relacionada con la salud (CAT) ha tenido un impacto alto previo alta hospitalaria COVID-19 y en cuanto a la relación entre el CAT y la mMRC se comprobó su dependencia, ya que aquellos pacientes con disnea grado 3 y 4 presentaban un impacto alto y muy alto en su calidad de vida.

\section{DISCUSIÓN}

$\mathrm{S}$ on escasos los estudios que evalúan la condición funcional respiratoria en pacientes post COVID-19 y sus secuelas, más aún si se trata de un abordaje desde el punto de vista de la fisioterapia; ya que recién se están levantando bases de datos para su análisis $\mathrm{y}$ estudio y pocos son los centros de médicos hospitalarios que permiten tener acceso a ellos.

Un informe del Centro Chino para el Control y Prevención de Enfermedades que incluyó 44.500 infecciones confirmadas con una estimación de la gravedad de la enfermedad, se informó de: cuadro Leve (sin neumonía o neumonía leve) un $81 \%$, Grave (Con disnea, hipoxia o> $50 \%$ de afectación pulmonar en la imagen en 24 a 48 horas) un $14 \%$, enfermedad crítica (Con insuficiencia respiratoria, shock o disfunción 
multiorgánica) el 5\%, la tasa general de letalidad fue de 2,3\%. Los pacientes que sobreviven a esta situación clínica presentan debilidad adquirida de paciente crítico, intolerancia al ejercicio, disfunción respiratoria y pérdida de calidad de vida (6).

Se puede esperar un comportamiento similar de la pandemia en el continente Americano; siendo sin lugar a dudas, mayor el impacto a nivel de países de Centro América y América del Sur, quienes en su mayoría presentan déficit a nivel de sus sistemas de salud y las consecuencias económicas a mediano y largo plazo podrían agravarse si la situación se prolonga en el tiempo.

En el presente estudio, se destaca la condición funcional respiratoria de pacientes Post Covid, valorando de una manera práctica e innovadora la disnea y su impacto en la calidad de vida mediante la utilización de medios virtuales frente a la emergencia mundial y sanitaria que causó el virus COVID-19. La cual también brinda oportunidad para acogernos a una rehabilitación a distancia donde se realicen sesiones filmadas para su tratamiento $\mathrm{y}$ seguimiento.

La Fisioterapia puede aminorar las nocivas complicaciones pulmonares respiratorias y musculo-esqueléticas que son comunes del COVID-19 en sus diferentes fases, similar al SARS en el 2003 (7), además el Fisioterapeuta no solo interviene en la evaluación, control y tratamiento de pacientes COVID-19 en hospitalización y en UCI, sino que también es piedra angular en el equipo interdisciplinar de salud, para la recuperación de las secuelas que esta enfermedad pueda dejar a nivel de función pulmonar y de capacidad funcional.

Como dato adicional a este estudio de evaluación, llamó la atención que el 33\% de la población evaluada manifestaron tener hormigueos o adormecimientos en pies y manos, el 51\% refirió debilidad muscular, así como dolor articular seguramente a consecuencia de la estadía prolongada en cama en las Unidades de Cuidados Intensivos "UCI".

Partiendo de los resultados obtenidos que podrán ser motivo de investigaciones posteriores es necesario entablar otros estudios como la fatiga y la relación de la misma en varios aspectos biopsicosocial, así como un conocimiento más profundo en otras índoles de la calidad de vida de pacientes Post COVID-19.

Esta situación de crisis mundial se presenta como una oportunidad para seguir un desarrollo profesional y científico, para implementar las ventajas que ofrecen las nuevas tecnologías en el ámbito de la telerehabilitación, así como para poner de manifiesto las carencias del Sistema Nacional de Salud en cuanto a las ratios de fisioterapeuta/paciente y la oportunidad para solventarlas desde los organismos competentes (8).

\section{CONCLUSIONES}

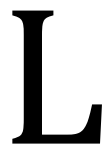
os datos obtenidos indican que tanto la edad, antecedentes patológicos y el tiempo de evolución previo a la hospitalización, tienen cierta influencia al momento de determinar el estado funcional actual de los pacientes COVID-19 después del alta hospitalaria, ya que de acuerdo a la base de datos de pacientes hospitalizados con COVID-19, del universo poblacional de 104 pacientes con COVID-19 y alta hospitalaria, 82 pacientes entre hombres y mujeres cumplían con los criterios de inclusión, siendo el sexo masculino el de mayor índice y el rango de edad entre de 50 y 59 años; donde la hipertensión 
sobresale entre las patologías preexistentes, seguida de la insuficiencia renal y la diabetes. La capacidad aeróbica de los pacientes en su mayoría es baja y guarda relación con el cansancio percibido según la escala de Borg entre moderado y grave; y un grado de disnea con similares características de moderada a severa.

De acuerdo a los resultados del cuestionario Assessment Test (CAT) de la calidad de vida y la salud, se pudo conocer que un porcentaje considerable de los pacientes indica que su calidad de vida ha sufrido un alto impacto posterior al contagio del COVID-19, pues la afectación constante a nivel pulmonar les impide desarrollar las actividades con la misma sencillez que antes.

En cuanto a la correlación entre la Disnea y el impacto de calidad de vida se comprobó su nivel de dependencia, indicando que mientras aumenta el grado de disnea sea esta moderada, severa o muy severa, el impacto de calidad de vida también aumenta (alto y muy alto), repercutiendo en el desarrollo de las actividades en la vida diaria, al contrario, si el grado de disnea es leve, el impacto de calidad de vida es bajo. Según los resultados del Inventario Breve de fatiga aquellos pacientes que refieren fatiga moderada o severa presentan mayor inferencia en su la calidad de vida.

El uso de los entornos virtuales se convierte en una excelente opción para apostar a un cambio en el ámbito laboral de la fisioterapia y de manera especial en el manejo de los pacientes post COVID-19, ya que por medio de la tele-rehabilitación se podrán dar las pautas necesarias de educación para el control del paciente a través de un familiar, lo cual es un gran reto para los profesionales de la Fisioterapia al crear y aplicar programas de actividad física e impartir estilos de vida saludable, que contribuyan a una mejor calidad de vida de los pacientes con COVID-19 y de la población en general.

- Conflicto de intereses. Los autores declaran no tener conflicto de intereses en la publicación de este artículo.

- Financiación. Autofinanciado

- Agradecimiento. Hospital General Monte Sinaí y Universidad Católica de Santiago de Guayaquil.

- Investigación realizada considerando los tratados bioéticos

\section{REFERENCIAS BIBLIOGRÁFICAS}

1. OMS. Protocolo de investigación de los primeros casos y sus contactos directos de la enfermedad por Coronavirus 2019 (COVID-19) [Internet]. 2020 [citado 21 de septiembre de 2020]. Disponible en: https://cutt.ly/clrpmNa

2. Ministerio de Salud Pública. Actualización de casos de coronavirus en Ecuador [Internet]. 2020 [citado 21 de septiembre de 2020]. Disponible en: https://www.salud.gob.ec/actualizacio n-de-casos-de-coronavirus-en-ecuador/

3. Dubé B-P, Vermeulen F, Laveneziana P. Disnea de esfuerzo en las enfermedades respiratorias crónicas: de la fisiología a la aplicación clínica. Arch Bronconeumol. 1 de febrero de 2017;53(2):62-70

4. Sepúlveda C V, Waissbluth A S, González GC, Sepúlveda CV, Waissbluth AS, González GC. Anosmia y enfermedad por Coronavirus 2019 (COVID-19): ¿Qué debemos saber? Rev Otorrinolaringol Cir Cabeza Cuello. Junio de 2020;80(2):247-58

5. Decaro N, Lorusso A. Novel human coronavirus (SARS-CoV-2): A lesson from animal coronaviruses. Vet Microbiol. Mayo de 2020;244:108693

6. Wu Z, McGoogan JM. Characteristics of and Important Lessons From the Coronavirus Disease 2019 (COVID-19) Outbreak in China: Summary of a 
Report of 72314 Cases From the Chinese Center for Disease Control and Prevention. JAMA. 7 de abril de 2020; 323(13):1239-42

7. Medicine $\mathrm{CA}$ of $\mathrm{R}$, Medicine $\mathrm{R}$ rehabilitation committee of $\mathrm{CA}$ of $\mathrm{R}$, Rehabilitation $\mathrm{C}$ rehabilitation G of CS of $\mathrm{PM}$ and. Recommendations for respiratory rehabilitation of COVID-19 in adult. Chin J Tuberc Respir Dis. 3 de marzo de 2020;43(00):E029-E029

8. Lista-Paz A, González-Doniz L, SoutoCamba S. ¿Qué papel desempeña la Fisioterapia en la pandemia mundial por COVID-19? Fisioterapia. Julio de 2020;42(4):167-9 